\title{
Knee injuries in severe trauma patients: a trauma registry study in 3.458 patients
}

\author{
Hagen Andruszkow ${ }^{1 *}{ }^{*}$, Emmanouil Liodakis $^{1 \dagger}$, Rolf Lefering ${ }^{2}$, Christian Krettek ${ }^{1}$, Frank Hildebrand ${ }^{1}$, Carl Haasper ${ }^{1}$ \\ and Trauma Registry of DGU ${ }^{3}$
}

\begin{abstract}
Background: Purpose of the presented study is to answer the following questions: Are knee injuries associated with trauma mechanisms or concomitant injuries? Do injuries of the knee region aggravate treatment costs or prolong hospital stay in polytraumatized patients?

Methods: A retrospective analysis including 29.779 severely injured patients (Injury Severity Score [greater than or equal to] 16) from the Trauma Registry of the German Society for Trauma Surgery database (1993-2008) was conducted. Patients were subdivided into two groups; the "Knee" group ( $n=3.458,11.6 \%$ of all patients) including all multiple trauma patients with knee injuries, and the "Non Knee" group $(n=26.321)$ including the remaining patients. Patients with knee injuries were slightly younger, less often male gender and had a significantly increased ISS.

Results: Patients in the Knee group suffered significantly more traffic accidents compared to the Non Knee group ( $82 \%$ vs. $52 \%, p<0.001$ ). These injuries were more often caused by car or motorbike accidents. Severe thoracic and limb injuries (AIS[greater than or equal to]3) were more frequently found in the Knee group $(p<0.001)$ while head injury was distributed equally. The overall hospital stay, ICU stay, and treatment costs were significantly higher for the Knee group (38.1 vs. 25.5 days, 15.2 vs. 11.4 days, 40,116 vs. 25,336 Euro, respectively; all $p<0.001$ ).

Conclusions: Traffic accidents are associated with an increased incidence of knee injuries than falls or attempted suicides. Furthermore, severe injuries of the limbs and chest are more common in polytraumatized patients with knee injuries. At last, treatment of these patients is prolonged and consequently more expensive.
\end{abstract}

\section{Background}

Knee injuries are commonly described in multiple traumatized ("polytraumatized") patients [1,2]. Although lower limb injuries and especially below knee injuries may not be life threatening apriori, they could cause significant functional disabilities with long lasting physical and psychosocial consequences [3-5]. Moreover, the increased risk of long-term sequels due to the complexity of knee injuries leads to relatively high financial costs $[4,6]$. Consequently, the socio-economic costs increase with the reduction of mortality of polytraumatized patients [7].

\footnotetext{
* Correspondence: andruszkow.hagen@mh-hannover.de

${ }^{\dagger}$ Equal contributors

${ }^{1}$ Trauma Department, Hannover Medical School, Carl-Neuberg-Straße 1,

Hannover 30625, Germany

Full list of author information is available at the end of the article
}

Despite the obvious relevance of lower limb injuries, they are commonly underestimated during the acute care setting while life threatening injuries or their complications such as sepsis or acute distress syndrome (ARDS) are treated [8]. To the best of our knowledge there are currently no studies designed to identify correlations between knee injuries and trauma mechanisms or concomitant injuries in patients suffering multiple trauma. This knowledge would lead physicians to focused search for knee injuries in high risk patients and may also influence the early initiation of treatment.

Therefore, the purpose of the presented study is to answer the following questions: (1) Do significant associations exist between knee injuries and trauma mechanisms or concomitant injuries? (2) Do injuries of the knee region aggravate treatment costs or prolong hospital stay in multiple trauma patients?

\section{Biomed Central}

(c) 2012 Andruszkow et al.; licensee BioMed Central Ltd. This is an Open Access article distributed under the terms of the Creative Commons Attribution License (http://creativecommons.org/licenses/by/2.0), which permits unrestricted use, distribution, and reproduction in any medium, provided the original work is properly cited. 


\section{Methods}

The Trauma Registry of the German Society for Trauma Surgery (TR-DGU)

The TR-DGU database is a multicenter, prospective, standardized and anonymous documentation of severely traumatized patients at four consecutive stages: A, prehospital phase; $\mathrm{B}$, emergency room and initial therapy until admission to intensive care unit (ICU); C, ICU phase; D, discharge from acute care hospital and outcome. Until 2008, 145 hospitals were affiliated with the TR-DGU, mostly from Germany $(n=128)$ [9]. The database contains detailed information on demographics, injury pattern, co-morbidities, pre-clinical and clinical management, time course, relevant laboratory findings and outcome of each individual. All data are centrally evaluated for completeness and reliability before entering into the database [8]. Injuries are coded according to the Abbreviated Injury Scale (AIS) following its 2005 revised version. The Trauma Registry is approved by the review board of the German Trauma Society (Deutsche Gesellschaft für Unfallchirurgie, DGU).

\section{Inclusion and exclusion criteria/ Classification of knee injuries}

All patients with an Injury Severity Score (ISS) $\geq 16$ documented in the TR-DGU from 1993-2008 were included in the present study.

Knee injuries were defined as all bony injuries of the patella, as well as soft tissue injuries involving the knee region with or without the involvement of articular cartilage. Distal femoral and proximal tibial fractures were considered as knee injuries as well.

Severity of injuries was recorded using the Abbreviated Injury Scale (AIS) as 1 (minor), 2 (moderate), 3 (severe, not life threatening), 4 (serious, life threatening), 5 (critical, survival uncertain), 6 (maximum, currently
Table 1 Demographic data

\begin{tabular}{lllll}
\hline & Knee & Non Knee & Total & p-value \\
\hline No. of patients & 3.458 & 26.321 & 29.779 & - \\
Gender (\% males) & $71.1 \%$ & $73.1 \%$ & $72.8 \%$ & 0.017 \\
Age (years) & $42.0 \pm 18.9$ & $43.0 \pm 20.8$ & $42.9 \pm 20.6$ & $<0.001$ \\
ISS & $31.0 \pm 11.9$ & $29.7 \pm 12.8$ & $29.9 \pm 12.7$ & $<0.001$ \\
\hline
\end{tabular}

The $\mathrm{p}$ value refers to the comparison of the Knee with the Non Knee group.

untreatable) [10]. However, the severity of knee injuries ranges only from one to three $[2,11]$.

\section{Statistics}

Treatment costs were based on a modular cost estimator based on data from the TR-DGU [12]. Continuous values are presented as mean \pm standard deviation (SD). Differences between the groups were evaluated with Student's t-test for continuous data, while Pearson's ChiSquare test was used for categorical values. A p-value $\leq$ 0.01 (two tailed) was considered to be statistically significant. Due to the large sample size statistical significance does not necessary imply clinical relevance. All statistical analyses were performed using SPSS (SPSS 18.0, IBM Inc., Somers, NY, USA).

\section{Results}

29.779 patients were included in the presented study and subsequently divided into two groups according to the presence of a knee injury. The group "Knee" includes 3.458 patients with any kind of knee injuries, and the group "Non Knee" includes the remaining 26.321 patients without a knee injury. In patients with severe trauma approximately every ninth case suffered from knee injuries $(n=3458,11.6 \%)$. Patients with knee injuries were slightly younger and had a significantly increased ISS (Table 1).

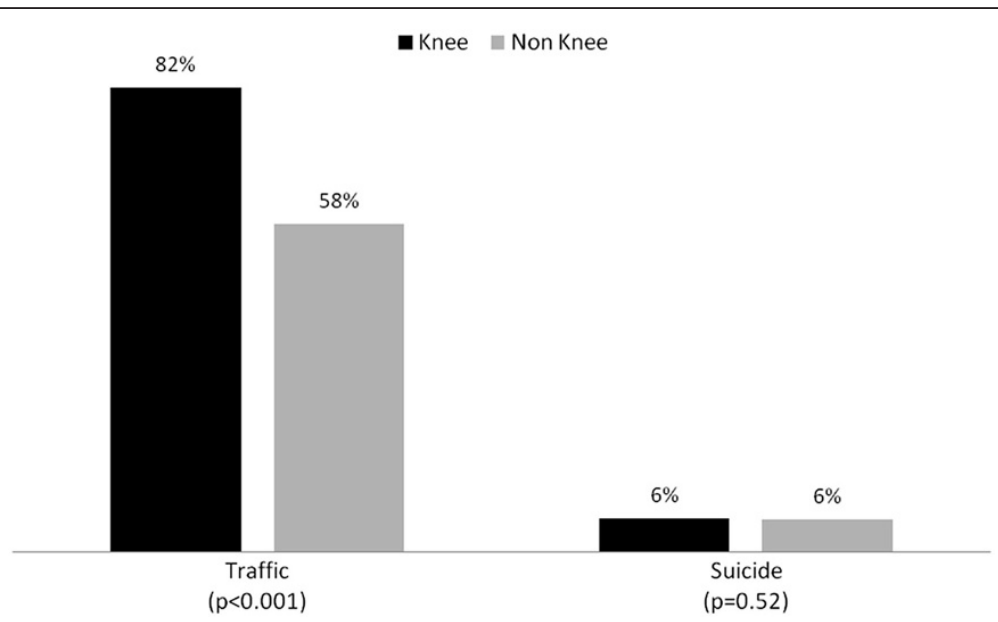

Figure 1 Knee injury and trauma mechanisms. 


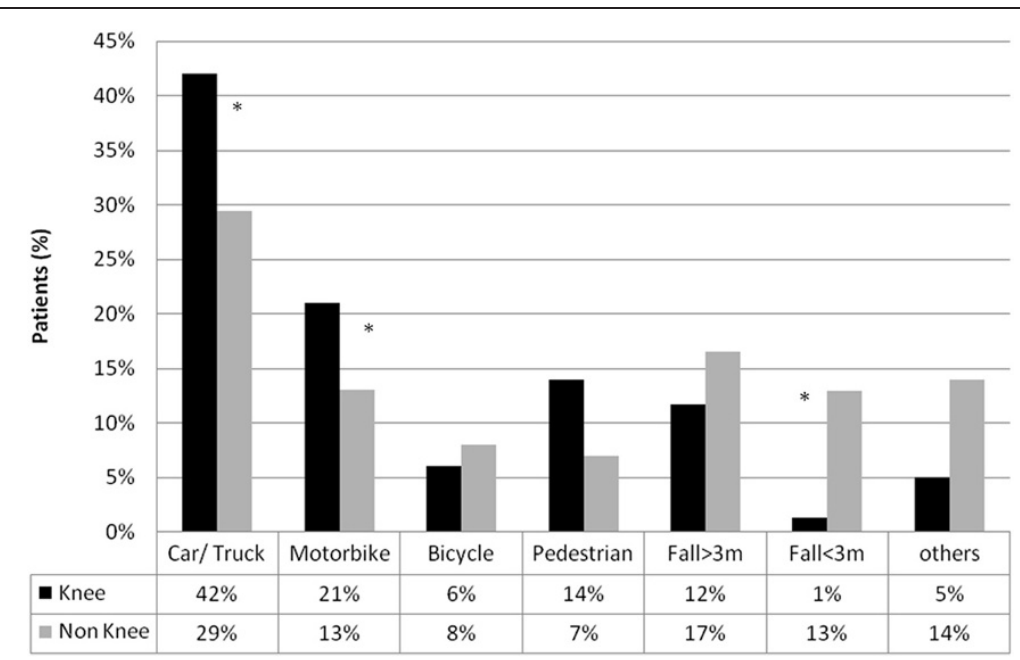

Figure 2 Details of injury mechanisms.

Concerning the mechanism of injury, knee injuries are significantly more often caused by traffic accidents compared to other reasons, e.g. suicide (Figure 1). Focusing on traffic accidents, knee injuries are more often associated with car/truck or motorbike accidents. Other traffic trauma mechanisms like bicycle or pedestrian accidents are less frequently associated with knee injuries (Figure 2). Furthermore, knee injuries seem to be seldom associated with low falls (Figure 2).

The injury pattern is documented by the incidence of severe concomitant injuries (AIS $\geq 3$ ) in different body regions (Figure 3). Knee injuries are associated with severe chest and limb injuries $(\mathrm{p}<0.001)$ and are more seldom described in patients with head trauma $(\mathrm{p}<0.001)$. Focusing patients with injuries of the lower extremities $(\mathrm{n}=14,305)$, the knee was involved in $24.2 \%$ cases. Analyzing the distribution of the knee injuries, the three most often observed diagnoses were patella fractures, cruciate ligament tears and proximal fractures of the tibia (all prevalence rates above 30\%, Table 2). Although traumatic knee dislocations are observed in only $4.4 \%$, this injury required many surgical procedures (1.7 operations per knee dislocation, Table 2, Figure 4).

As far as the outcome of both groups is concerned, the Knee group required on average about 4 more days on ICU and 13 more days in the acute care hospital (Table 3). Accordingly, costs were significantly increased in the Knee group (40,116 vs. 25,336 Euro, respectively; $\mathrm{p}<0.001$; Table 3). Mortality was $9.3 \%$ for the Knee group and $19.3 \%$ for the Non Knee group $(\mathrm{p}<0.001)$.

\section{Discussion}

Since hospital mortality of multiple injured patients is decreasing during the past decades, the relevance of early detection and initiation of adequate treatment in patients with knee injuries increases [7]. Following the

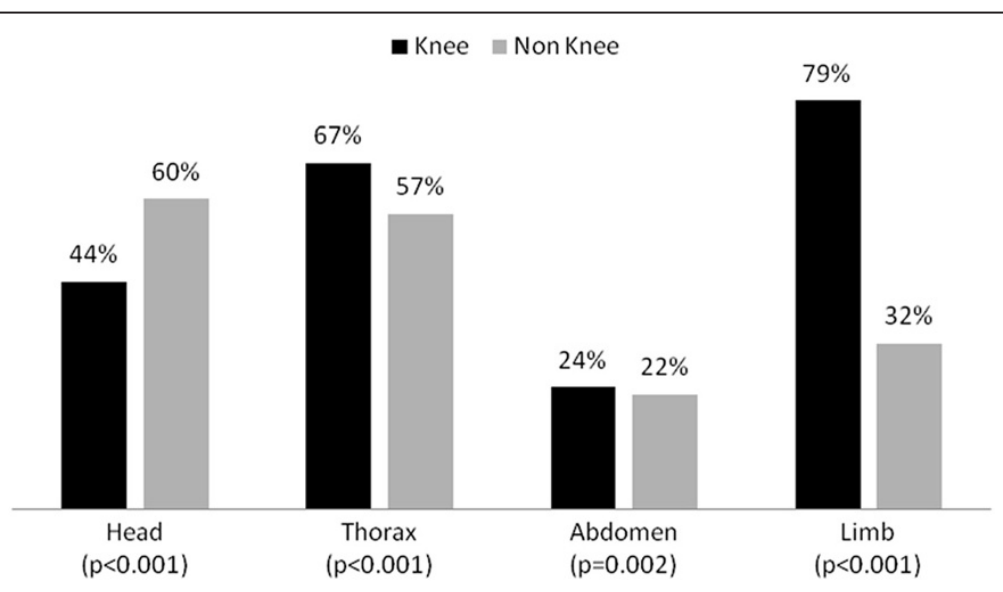

Figure 3 Association of knee injuries with severe (AIS severity $\geq 3$ ) concomitant injuries of other body regions. 
Table 2 Distribution of injuries of the knee region and mean number of operations per diagnosis in patients that required at least one operation

\begin{tabular}{lcc}
\hline Diagnosis & $\begin{array}{l}\text { No. of operations } \\
\text { per diagnosis }\end{array}$ & $\begin{array}{c}\text { Prevalence } \\
\text { of injury }\end{array}$ \\
\hline Patellar tendon rupture & 1.5 & $4.1 \%$ \\
Patella fracture & 1.3 & $37.1 \%$ \\
Knee dislocation & 1.7 & $4.4 \%$ \\
Cruciate ligament tear & 1.5 & $38.0 \%$ \\
Femur fracture & 1.9 & $18.7 \%$ \\
Tibia fracture & 2.0 & $32.9 \%$ \\
Other knee injury & 1.4 & $16.4 \%$ \\
\hline
\end{tabular}

This analysis is based on 4.388 different diagnoses in the Knee group.

presented results, $90.7 \%$ of all patients with knee injuries were discharged alive. The majority of this population is constituted by young patients (42.0 years old), who have to deal with potential functional knee disabilities such as instability, reduced range of motion or pain for many decades. Additionally, endoprosthetic replacement in this young population presents usually only compromise and not permanent solution. Identification of patients at risk for injuries in the knee region may help to decrease late complications.

According to our data, traffic accidents with car/truck or motorbike result more commonly in knee injuries than suicide attempts or falls. A recent German accident investigation study analyzed more than 40,000 victims of road traffic accidents from 1985 to 2003 [1,2]. In accordance to our results, the authors demonstrated an increased knee injury rate in motorbike and car or truck patients. Contrary to our study, most patients were not severely injured (ISS $<16$ ). A reduction of knee injury prevalence was observed during this period probably because of evolution in car design and the increased use of protective pads and clothes developed for motorcyclists. However, identifying the precise mechanism of knee injury in traffic accidents was not possible, as many components seemed to interact. Severe lower limb injuries of bicyclists or pedestrians are usually seen in collision with cars [13]. The most common injury is the proximal tibia fracture.

Jumps or falls from a height greater than $3 \mathrm{~m}$ cause usually fractures in the thoracolumbar spine, ankle and foot. Thereby, fractures of the ankle and the calcaneus are most commonly described [14]. The small incidence of knee injuries in this population may base upon three possible explanations: Firstly, as jumpers jump foot first for a suicide, there is usually no direct impact on the patella [15]. The second reason may be found in the absence of rotation-varus/ valgus forces that put into stress the collateral and cruciate ligaments. Finally, severe injuries (ISS $\geq 16$ ) are usually induced by falls from a height less than $3 \mathrm{~m}$ if the person falls with the head first.

Most common injuries observed in our study are patella fractures caused by direct impact on the knee and cruciate ligament ruptures caused by rotation-varus/ valgus stress mechanisms. Although patellar tendon ruptures are seldom represented (4.1\%), this injury requires surgery very often (86\%). Etiology of these ruptures in young individuals without risk factors such as corticosteroid use, rheumatoid diseases or diabetes was usually direct trauma with or without open wounds in that region [16].

As far as treatment of injuries of the knee region is concerned, we observe that $38 \%$ of knee dislocations, $45 \%$ of meniscus tears and $66 \%$ of collateral and cruciate ligament injuries were treated non-operatively. Possible explanations for these unexpected results cannot be elucidated by our database. However, based on the

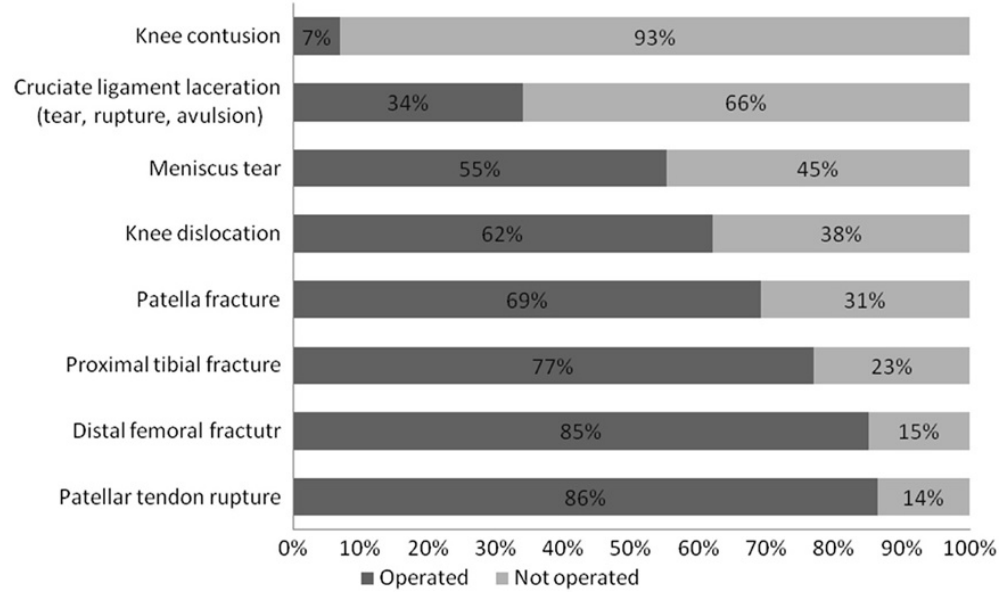

Figure 4 Management strategies of knee injuries. 
Table 3 Outcome of patients with and without knee injury

\begin{tabular}{lllll}
\hline & Knee & Non Knee & Total & p-value \\
\hline Overall hospital stay (days) & $38.1 \pm 33.0$ & $25.5 \pm 29.1$ & $26.9 \pm 29.9$ & $<0.001$ \\
ICU stay (days) & $15.2 \pm 16.8$ & $11.4 \pm 14.3$ & $11.8 \pm 14.6$ & $<0.001$ \\
Intubation duration (days) & $9.9 \pm 14.2$ & $7.4 \pm 11.6$ & $7.7 \pm 12.0$ & $<0.001$ \\
Mortality & $14.5 \%$ & $17.4 \%$ & $16.7 \%$ & $<0.001$ \\
Total costs of treatment (Euro) & $40,166 \pm 28,266$ & $25,336 \pm 22,750$ & $27,087 \pm 23,951$ & $<0.001$ \\
\hline
\end{tabular}

high injury severity of the patients included we believe that potential damage control or intensive care treatment determined the decision for non-surgical treatment [17].

Referring to the main aspects of treatment duration as well as primary outcome, it has to be stated that patients with knee injuries were slightly younger, less often male gender and had suffered a higher injury severity in the present study. However, analyzing the extent of differences focusing age and gender distribution, this aspect seems actually negligibly. Nevertheless, one might argue that increased treatment durations as well as costs might result solely from the increased injury severity without impact of "knee" influences. But as the differences of injury distribution seem descriptively minor (31.0 vs. 29.7), these differences might be argued as minor clinical relevance. This argument is supported by the presented mortality demonstrating an approximately $10 \%$ higher mortality in the Non Knee group despite the decreased injury severity. In addition, a statistical difference of three points ISS has already been accepted as less clinically relevant [18]. Another aspect mentioned is found in the injury severity score itself. In patients with multiple injuries confined to a single body region, the ISS considers only one of the injuries within that region [19]. In effect, the ISS ignores all but the worst injury per body region, which could result in a considerable underestimation in case of isolated extremity injuries [19].

However, treatment durations are influenced by many factors which are certainly not elucidated entirely by the presented study, although the presented register data enables vast data analyses. Nevertheless, we feel safe to argue the knee injury as one aspect to impact treatment durations and costs beside the injury severity and demographic parameters.

In conclusion, traffic accidents are associated with a higher incidence of knee injuries than falls or attempted suicides. Severe limb and chest injuries are more commonly found in polytraumatized patients with knee injuries. At last, treatment of these patients is prolonged and costs of treatment are higher.

\section{Competing interests}

Each author certifies that he has no commercial associations (e.g., consultancies, stock ownership, equity interest, etc.) that might pose a conflict of interest in connection with the submitted article.

\section{Authors' contributions}

$\mathrm{HA}$ and EL conceived this study designing the trial, provided statistical advice on study design, analyzed the data and drafted the manuscript. HA and EL have been contributed equally to the writing of this manuscript. RL provided statistical advice on the study design, analyzed the data and supervised the conduct of the trial and data collection. RL, CK, FH and $\mathrm{CH}$ conceived the study and designed the trial. FH conceived the study, designed the trial, and supervised the conduct of the trial. $\mathrm{CH}$ conceived the study, designed the trial, supervised the conduct of the trial and data collection, provided statistical advice on study design and analyzed the data. HA takes responsibility for the article as a whole. All authors contributed substantially to manuscript revision. All authors have read and approved the final manuscript for publication.

\section{Author details}

${ }^{1}$ Trauma Department, Hannover Medical School, Carl-Neuberg-Straße 1, Hannover 30625, Germany. ${ }^{2}$ Institute for Research in Operative Medicine (IFOM), Cologne-Merheim Medical Center, University Witten-Herdecke, Cologne, Germany. ${ }^{3}$ Committee on Emergency Medicine, Intensive and Trauma Care of the German Society for Trauma Surgery (DGU), Cologne, Germany.

Received: 8 December 2011 Accepted: 23 July 2012 Published: 6 August 2012

\section{References}

1. Haasper C, Otte D, Knobloch K, Zeichen J, Krettek C, Richter M: Knee injuries of vulnerable road users in road traffic. Unfallchirurg 2006, 109:1025-1031.

2. Haasper C, Otte D, Knobloch K, Probst C, Board TN, Krettek C, Richter M: Knee injuries in restrained car drivers in German road traffic accidents. J Trauma 2008, 65:136-141

3. Levy BA, Marx RG: Outcome after knee dislocation. Knee Surg Sports Traumatol Arthrosc 2009, 17:1011-1012.

4. Siegel JH, Mason-Gonzalez S, Dischinger PC, Read KM, Cushing BM, Badellino MC, Goodarzi S, Smialek JE, Heatfield BM, Robinson RM, et al: Causes and costs of injuries in multiple trauma patients requiring extrication from motor vehicle crashes. J Trauma 1993, 35:920-931.

5. Zelle BA, Brown SR, Panzica M, Lohse R, Sittaro NA, Krettek C, Pape HC: The impact of injuries below the knee joint on the long-term functional outcome following polytrauma. Injury 2005, 36:169-177.

6. Dischinger PC, Read KM, Kufera JA, Kerns TJ, Burch CA, Jawed N, Ho SM, Burgess AR: Consequences and costs of lower extremity injuries. Annu Proc Assoc Adv Automot Med 2004, 48:339-353.

7. Probst C, Pape HC, Hildebrand F, Regel G, Mahlke L, Giannoudis P, Krettek C, Grotz MR: 30 years of polytrauma care: An analysis of the change in strategies and results of 4849 cases treated at a single institution. Injury 2009, 40:77-83.

8. Probst C, Richter M, Lefering R, Frink M, Gaulke R, Krettek C, Hildebrand F: Incidence and significance of injuries to the foot and ankle in polytrauma patients-an analysis of the Trauma Registry of DGU. Injury 2010, 41:210-215.

9. Paffrath T, Wafaisade A, Lefering R, Simanski C, Bouillon B, Spanholtz T, Wutzler S, Maegele M: Venous thromboembolism after severe trauma: incidence, risk factors and outcome. Injury 2010, 41:97-101.

10. Garthe E, States JD, Mango NK: Abbreviated injury scale unification: the case for a unified injury system for global use. J Trauma 1999, 47:309-323. 
11. Torjussen J, Bahr R: Injuries among elite snowboarders (FIS Snowboard World Cup). Br J Sports Med 2006, 40:230-234.

12. Pape HC, Grotz M, Schwermann T, Ruchholtz S, Lefering R, Rieger M, Troger $M$, von der Schulenburg JM G, Krettek C: The development of a model to calculate the cost of care for the severely injured-an initiative of the Trauma Register of the DGU. Unfallchirurg 2003, 106:348-357.

13. Richter M, Otte D, Haasper C, Knobloch K, Probst C, Westhoff J, Sommer K, Krettek C: The current injury situation of bicyclists-a medical and technical crash analysis. J Trauma 2007, 62:1118-1122.

14. Richter D, Hahn MP, Ostermann PA, Ekkernkamp A, Muhr G: Vertical deceleration injuries: a comparative study of the injury patterns of 101 patients after accidental and intentional high falls. Injury 1996, 27:655-659.

15. Teh J, Firth M, Sharma A, Wilson A, Reznek R, Chan O: Jumpers and fallers: a comparison of the distribution of skeletal injury. Clin Radiol 2003, 58:482-486

16. Amlang $\mathrm{MH}$, Zwipp H: Damage to large tendons: Achilles, patellar and quadriceps tendons. Chirurg 2006, 77:637-649. quiz 649.

17. Hildebrand F, Giannoudis P, Kretteck C, Pape HC: Damage control: extremities. Injury 2004, 35:678-689.

18. Hoffmann M, Lefering R, Gruber-Rathmann M, Rueger JM, Lehmann W: The impact of BMI on polytrauma outcome. Injury 2012, 43:184-188.

19. Osler T, Baker SP, Long W: A modification of the injury severity score that both improves accuracy and simplifies scoring. J Trauma 1997, 43:922-925. discussion 925-926.

doi:10.1186/1752-2897-6-7

Cite this article as: Andruszkow et al:: Knee injuries in severe trauma patients: a trauma registry study in 3.458 patients. Journal of Trauma Management \& Outcomes 2012 6:7.

\section{Submit your next manuscript to BioMed Central and take full advantage of:}

- Convenient online submission

- Thorough peer review

- No space constraints or color figure charges

- Immediate publication on acceptance

- Inclusion in PubMed, CAS, Scopus and Google Scholar

- Research which is freely available for redistribution 